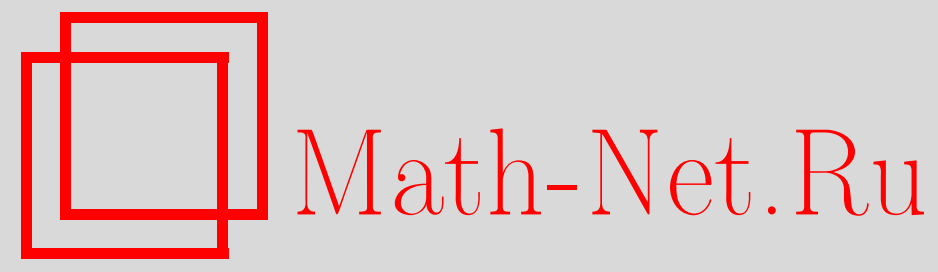

М. А. Власенко, А. С. Меллит, Ю. С. Самойленко, Об алгебрах, порожденных линейно связанными образующими с заданным спектром, Функи. анализ и его прил., 2005, том 39, выпуск 3, 14-27

DOI: https://doi.org/10.4213/faa71

Использование Общероссийского математического портала Math-Net.Ru подразумевает, что вы прочитали и согласны с пользовательским соглашением

http://www . mathnet.ru/rus/agreement

Параметры загрузки:

IP: 54.174.149.18

26 апреля 2023 г., 13:22:32

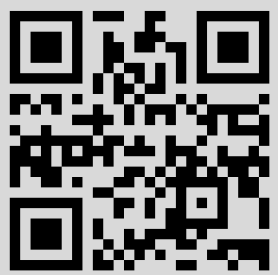




\title{
Об алгебрах, порожденных линейно связанными образующими с заданным спектром*
}

\author{
(C) 2005. М. А. ВЛАСенко, А. С. МЕЛЛИТ, Ю. С. САМОЙЛЕнко
}

\section{Введение}

Наборы операторов $\left\{X_{k}\right\}_{k=1}^{N}$ в сепарабельном комплексном гильбертовом пространстве $\mathbb{H}$, спектры $\sigma\left(X_{k}\right)$ которых принадлежат заданным конечным множествам $M_{k} \subset \mathbb{C}(k=1, \ldots, N)$ и сумма которых скалярна,

$$
\sum_{k=1}^{N} X_{k}=\lambda \mathbb{I}_{\mathbb{H}}
$$

играют важную роль в анализе, алгебраической геометрии, теории операторов, математической физике (см. $[1,2]$ и приведенную там библиографию).

В настоящей работе изучается соответствующий алгебраический объект. Все рассматриваемые алгебры являются ассоциативными алгебрами над полем комплексных чисел с единицей, а все рассматриваемые полиномы - полиномами от одной переменной над полем комплексных чисел. Пусть $P_{1}, \ldots, P_{N}-$ полиномы степени не меньше 2. Рассмотрим следующую алгебру с $N$ образующими:

$$
A_{P_{1}, \ldots, P_{N}}=\mathbb{C}\left\langle x_{1}, \ldots, x_{N} \mid P_{i}\left(x_{i}\right)=0 \quad \forall i, \sum_{i=1}^{N} x_{i}=0\right\rangle
$$

Для всевозможных наборов полиномов $P_{1}, \ldots, P_{N}$ мы изучаем вопрос, являются ли алгебры $A_{P_{1}, \ldots, P_{N}}$ конечномерными, и находим их рост в бесконечномерном случае (§2). О понятии роста и порядка роста см. [3]. Оказывается, что ответ зависит лишь от набора степеней полиномов $\operatorname{deg} P_{1}, \ldots, \operatorname{deg} P_{N}$. В $\S 3$ изучается наличие полиномиальных тождеств в этих алгебрах.

Пусть $k_{1}, \ldots, k_{N}$ - некоторые натуральные числа, большие 1. Обозначим через $T_{k_{1}, \ldots, k_{N}}$ неориентированный граф, представляющий собой $N$ ветвей с $k_{1}, \ldots, k_{N}$ вершинами, склеенных в одной вершине. Например,

$$
T_{3,2,2}=
$$

Поставим в соответствие алгебре $A_{P_{1}, \ldots, P_{N}}$ граф $\mathbb{G}=T_{\operatorname{deg} P_{1}, \ldots, \operatorname{deg} P_{N}}$. Параметризуем алгебры, которым соответствует данный граф $\mathbb{G}$, наборами корней полиномов $P_{1}, \ldots, P_{N}$ с учетом кратностей. В $\S 2$ статьи установлен следующий результат:

*Эта работа частично поддержана грантом DFG 436 UKR 113/71 (Германия) и грантом Государственного фонда фундаментальных исследований Украины № 01.07/071. 
Теорема 1. (1) Если $\mathbb{G}$ - диаграмма Дынкина, то алгебра $A_{P_{1}, \ldots, P_{N}}$ конечномерна и ее размерность не превышает $2(n+1)$ для $D_{n}, 12$ для $E_{6}, 24$ для $E_{7}$ и 60 для $E_{8}$. Оиенки достигаются для алгебр с нильпотентными образующими (m. е. когда $\left.P_{i}(x)=x^{k_{i}}, 1 \leqslant i \leqslant N\right)$. Существует конечный набор гиперплоскостей в пространстве параметров (т.е. корней полиномов), вне которого все соответствующие алгебры нулевые.

(2) Если $\mathbb{G}$ - расширенная диаграмма Дьнкина, то алгебра $A_{P_{1}, \ldots, P_{N}}$ имеет квадратичный порядок роста.

(3) Если граф $\mathbb{G}$ не является ни диаграммой Дынкина, ни расширенной диаграммой Дьнкина, то алгебра $A_{P_{1}, \ldots, P_{N}}$ содержсит свободную подалгебру с двумя образующими.

Все конечномерные алгебры являются алгебрами с полиномиальными тождествами, т. е. РІ-алгебрами (о РІ-алгебрах см. например, [4, 5, 3]). Все РІ-алгебры имеют не более чем полиномиальный рост. Рассмотрим семейство алгебр, которым соответствуют расширенные диаграммы Дынкина. Свойство таких алгебр быть PI-алгебрами уже зависит от самих полиномов, а не только от их степеней. Пусть $S=S\left(P_{1}, \ldots, P_{N}\right)$ задается равенством $S=\sum_{i=1}^{N} s_{i}$, где $s_{i}$ есть среднее арифметическое корней полинома $P_{i}$ с учетом их кратностей.

ГиПотезА 1. Алгебра $A_{P_{1}, \ldots, P_{N}}$, для которой граф $\mathbb{G}=T_{\operatorname{deg} P_{1}, \ldots, \operatorname{deg} P_{N}}$ есть расширенная диаграмма Дьнкина, является РІ-алгеброй тогда и только тогда, когда $S\left(P_{1}, \ldots, P_{N}\right)=0$, причем если $\mathbb{G}=\widetilde{D}_{4}$, то это $\mathbb{F}_{4}$-алгебра, а в случае $\widetilde{E}_{6}, \widetilde{E}_{7}$ и $\widetilde{E}_{8}$ - соответственно $\mathbb{F}_{6}-, \mathbb{F}_{8}-$ и $\mathbb{F}_{12}$-алгебра.

Напомним, что $\mathbb{F}_{n}$-алгеброй называется алгебра, в которой для произвольных элементов $y_{1}, \ldots, y_{n}$ выполняется тождество $\sum_{\sigma \in S_{n}} \operatorname{sgn} \sigma y_{\sigma(1)} \ldots y_{\sigma(n)}=0$.

Для $\widetilde{D}_{4}$ гипотеза 1 доказана в [6,7]. Для $\widetilde{E}_{6}$ при $P_{1}(x)=P_{2}(x)=P_{3}(x)=$ $(x-1) x(x+1)$ в работе [8] с помощью результатов из [9] показано, что $A_{P_{1}, P_{2}, P_{3}}$ является $\mathbb{F}_{6}$-алгеброй.

В $\S 3$ настоящей работы мы доказываем достаточность условия гипотезы (теорема 2), т. е. показываем, что для расширенных диаграмм Дынкина алгебры $A_{P_{1}, \ldots, P_{N}}$ при $S=0$ являются РІ-алгебрами.

Рассматриваемые алгебры тесно связаны с деформированными препроективными алгебрами, введенными в [10]. Пусть $Q$ - некоторый колчан, а $Q_{0}$ и $Q_{1}-$ множества его вершин и стрелок соответственно. Рассмотрим удвоенный колчан $\bar{Q}$, полученный из $Q$ добавлением для каждой стрелки $a: i \rightarrow j, a \in Q_{1}$, новой стрелки $a^{*}: j \rightarrow i$. Пусть $\lambda: Q_{0} \rightarrow \mathbb{C}-$ некоторая функция. Тогда деформированная препроективная алгебра есть

$$
\Pi^{\lambda}(Q)=\mathbb{C}(\bar{Q}) /\left(\sum_{a \in Q_{1}}\left[a, a^{*}\right]-\sum_{i \in Q_{0}} \lambda(i) e_{i}\right)
$$

где $\mathbb{C}(\bar{Q})$ - алгебра путей колчана $\bar{Q}$, а $e_{i}$ - идемпотент, соответствующий вершине $i \in Q_{0}$. Препроективные алгебры $\Pi^{0}(Q)$ изучались многими авторами (см. [11] и приведенную там библиографию). Близкие алгебры ранее рассматривали в [12] И. М. Гельфанд и В. А. Пономарев.

Пусть $\alpha_{0}^{i}, \alpha_{1}^{i}, \ldots, \alpha_{\operatorname{deg} P_{i}-1}^{i}$ - корни полинома $P_{i}$. Ориентируем ребра графа $\mathbb{G}$ по направлению к центральной вершине и рассмотрим функцию $\lambda$ на вершинах 
этого графа, которая для $i$-й ветви определяется следующим образом:

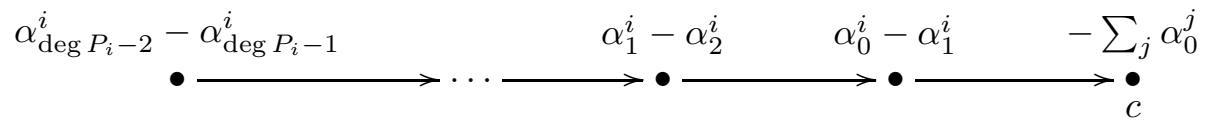

где $c \in \mathbb{G}_{0}$ - центральная вершина.

Справедливо следующее

ПРЕДЛОЖЕНИЕ 1. Алгебри $A_{P_{1}, \ldots, P_{N}} \quad u \quad e_{c} \Pi^{\lambda}(\mathbb{G}) e_{c}$ изоморфнъ. Алгебра $A_{P_{1}, \ldots, P_{N}}$ конечномерна тогда и только тогда, когда конечномерна алгебра $\Pi^{\lambda}(\mathbb{G})$. Если эти алгебры бесконечномерны, они имеют одинаковыи порядок pocma.

Таким образом результаты данной работы о конечномерности и росте алгебр $A_{P_{1}, \ldots, P_{N}}$ можно получить из работ [10] и [12], а наличие полиномиальных тождеств в $A_{P_{1}, \ldots, P_{N}}$ - из [10], используя предложение 1. Однако наши методы отличны от методов указанных работ, теорема 1 содержит точные оценки размерностей алгебр для графов Дынкина и в теореме 2 утверждается конечномерность над центром алгебр $A_{P_{1}, \ldots, P_{N}}$ при $S\left(P_{1}, \ldots, P_{N}\right)=0$ для расширенных диаграмм Дынкина.

Отметим, что алгебры, порожденные линейно связанными проекторами, представления которых рассматривались в работах $[13,14]$, являются частным случаем алгебр $A_{P_{1}, \ldots, P_{N}}$. Кроме того, с помощью утверждения, аналогичного утверждению 1 , можно установить связь алгебр $A_{P_{1}, \ldots, P_{N}}$ с локально скалярными представлениями графов (см. [15]).

\section{§1. Предварительные сведения}

В этой работе мы рассматриваем алгебры с единицей над полем комплексных чисел $\mathbb{C}$.

Пусть $\mathscr{F}=\mathbb{C}\left\langle x_{1}, \ldots, x_{N}\right\rangle$ - свободная алгебра, $W$ - множество слов в алфавите $x_{1}, \ldots, x_{N}$, и пусть $I=I\left(F_{1}, \ldots, F_{M}\right)$ - идеал, порожденный элементами $F_{1}, \ldots, F_{M} \in \mathscr{F}$. На множестве образующих зададим порядок $x_{1}<\cdots<x_{N}$. Ему соответствует градуированный лексикографический порядок на $W$ : бо́льшим считается слово большей длины, а при равной длине то, у которого на первой различающейся позиции стоит бо́льшая образующая. Слово называется нормальным, если в алгебре $\mathscr{F} / I$ оно не является линейной комбинацией меньших слов. Нормальные слова образуют линейный базис в $\mathscr{F} / I$. Кроме того, нормальные слова длины $\leqslant k$ образуют базис в пространстве, порожденном словами длины $\leqslant k$.

Пусть $F\left(x_{1}, \ldots, x_{N}\right)$ - некоторый полином. Его главной частъю будем называть полином $\widehat{F}\left(x_{1}, \ldots, x_{N}\right)$, состоящий из мономов старшей степени полинома $F$. В дальнейшем нам понадобится

Лемма 1. Пусть $A=\mathbb{C}\left\langle x_{1}, \ldots, x_{N} \mid F_{i}\left(x_{1}, \ldots, x_{N}\right)=0, i=1, \ldots, M\right\rangle-$ некоторая конечно заданная алгебра. Тогда размерность (рост) алгебры $A^{0}=$ $\mathbb{C}\left\langle x_{1}, \ldots, x_{N} \mid \widehat{F}_{i}\left(x_{1}, \ldots, x_{N}\right)=0, i=1, \ldots, M\right\rangle$ не менъше размерности (роста) алгебры $A$.

ДокАЗАтельство. Достаточно показать, что если некоторое слово $w$ не является нормальным в алгебре $A^{0}$, то оно не является нормальным и в алгебре $A$. 
Рассмотрим разложение

$$
w=\sum_{v<w} C_{v} v \quad \text { в алгебре } A^{0},
$$

где $C_{v}(v \in W)$ - некоторые комплексные коэффициенты. Значит, для некоторого набора комплексных чисел $\left(D_{v_{1} v_{2}}^{i}\right)$

$$
w=\sum_{v<w} C_{v} v+\sum_{i=1}^{M} \sum_{v_{1}, v_{2} \in W} D_{v_{1} v_{2}}^{i} v_{1} \widehat{F}_{i} v_{2} \quad \text { в свободной алгебре } \mathscr{F},
$$

причем можно считать, что в последней сумме присутствуют лишь такие слагаемые, в которых порядок однородного полинома $v_{1} \widehat{F}_{i} v_{2}$ не превышает длины слова $w$. Действительно, из равенства (1.1) следует, что сумма всех других слагаемых равна нулю, так что их можно исключить. Значит,

$$
w=\sum_{v<w} C_{v} v+\sum_{i=1}^{M} \sum_{v_{1}, v_{2}} D_{v_{1} v_{2}}^{i} v_{1}\left(\widehat{F}_{i}-F_{i}\right) v_{2} \quad \text { в алгебре } A
$$

и поскольку порядок полинома $\widehat{F}_{i}-F_{i}$ меньше порядка однородного полинома $\widehat{F}_{i}$ для каждого $i$, порядок каждого слагаемого в последней сумме меньше длины слова $w$. Тогда (1.2) и есть требуемое разложение.

Алгоритмы построения множества нормальных слов и вычисления порядка роста конечно заданной алгебры хорошо известны (см., например, [3]). Базисом Грёбнера идеала $I$ называется множество $\operatorname{Gr} \subset I$ элементов этого идеала, такое, что для всякого элемента $g \in I$ его старшее слово содержит в качестве подслова одно из старших слов элементов множества Gr. Базис Грёбнера $\mathrm{Gr}$ называется минималъным, если никакое его собственное подмножество не является базисом Грёбнера. Слово является нормальным в факторалгебре $\mathscr{F} / I$ тогда и только тогда, когда оно не содержит в качестве подслова старшего слова какого-нибудь элемента базиса Грёбнера. Существует алгоритм построения минимального базиса Грёбнера в идеале $I=I\left(F_{1}, \ldots, F_{M}\right)$, исходя из элементов $F_{1}, \ldots, F_{M}$. Этот алгоритм не обязательно заканчивает работу за конечное число шагов. Существует ли конечный базис Грёбнера, вообще говоря, зависит от исходного выбора порядка на образующих.

Если у нас есть конечный базис Грёбнера, то вычисление роста алгебры сводится к вычислению роста специального ориентированного графа. Вершинами графа роста алгебры являются все нормальные слова длины, меньшей на 1, чем максимальная длина старшего слова элементов в базисе Грёбнера. Ребро от слова $w$ к слову $v$ существует, если и только если для некоторых образующих $x_{i}$ и $x_{j}$ (возможно, совпадающих) слова $w x_{i}$ и $x_{j} v$ равны и нормальны.

\section{§2. Размерность и рост алгебр}

Зафиксируем набор степеней полиномов $k_{1}, \ldots, k_{N}$, т. е. граф $\mathbb{G}=T_{k_{1}, \ldots, k_{N}}$. Обозначим символом $\mathbb{M}_{\mathbb{G}}$ семейство всех алгебр $A_{P_{1}, \ldots, P_{N}}$, где $\operatorname{deg} P_{i}=k_{i}$ для Bcex $i$. 
ТАБлицА 1. Конечномерные алгебры

\begin{tabular}{|c|c|c|c|c|}
\hline$D_{n}$ & $\begin{array}{l}x_{1}^{n-2}=x_{2}^{2}=x_{3}^{2}=0 \\
x_{1}+x_{2}+x_{3}=0 \\
x_{3}>x_{2}>x_{1}\end{array}$ & $\begin{array}{l}\frac{x_{3}}{x_{2}}+x_{2}+x_{1}, \\
\frac{x_{2}^{2}}{\frac{x_{1}^{n}}{2}-2} x_{2}+x_{1}^{2}, \\
\underline{ }\end{array}$ & $\begin{array}{l}1, x_{1}, \ldots, x_{1}^{n-3} \\
x_{2}, x_{1} x_{2}, \ldots, x_{1}^{n-3} x_{2}\end{array}$ & $2(n-2)$ \\
\hline$E_{6}$ & $\begin{array}{l}x_{1}^{3}=x_{2}^{3}=x_{3}^{2}=0 \\
x_{1}+x_{2}+x_{3}=0 \\
x_{3}>x_{2}>x_{1}\end{array}$ & $\begin{array}{l}\frac{x_{3}}{x_{2}^{2}}+x_{2}+x_{1}, \\
\frac{x_{2}}{x_{1} x_{2}}-x_{1}+x_{1} x_{2} x_{1}, x_{1}^{2}, \\
\frac{x_{1}^{3}}{x_{2}} x_{1}^{2} x_{2}+x_{1} x_{2} x_{1}^{2}+x_{1}^{2} x_{2} x_{1}\end{array}$ & $\begin{array}{l}1, x_{1}, x_{2}, \\
x_{1}^{2}, x_{1} x_{2}, x_{2} x_{1}, \\
x_{1}^{2} x_{2}, x_{1} x_{2} x_{1}, x_{2} x_{1}^{2}, \\
x_{1} x_{2} x_{1}^{2}, x_{1}^{2} x_{2} x_{1}, \\
x_{1}^{2} x_{2} x_{1}^{2}\end{array}$ & 12 \\
\hline$E_{7}$ & $\begin{array}{l}x_{1}^{4}=x_{2}^{3}=x_{3}^{2}=0 \\
x_{1}+x_{2}+x_{3}=0 \\
x_{3}>x_{2}>x_{1}\end{array}$ & $\begin{array}{l}\frac{x_{3}}{x_{2}^{2}}+x_{2}+x_{1}, \\
\frac{x_{2} x_{1} x_{2}}{x_{1}^{4}}-x_{1} x_{2} x_{1}-x_{1}^{3}, \\
\frac{x_{2} x_{1}^{2} x_{2}}{x_{2} x_{1}^{3} x_{2}} x_{1}+x_{2} x_{1}^{3}+\ldots,\end{array}$ & $\begin{array}{l}1, x_{1}, x_{2}, \\
x_{1}^{2}, x_{1} x_{2}, x_{2} x_{1}, \\
x_{1}^{3}, x_{1}^{2} x_{2}, \ldots, \\
\ldots\end{array}$ & 24 \\
\hline$E_{8}$ & $\begin{array}{l}x_{1}^{5}=x_{2}^{3}=x_{3}^{2}=0 \\
x_{1}+x_{2}+x_{3}=0 \\
x_{3}>x_{2}>x_{1}\end{array}$ & $\begin{array}{l}\frac{x_{3}}{x_{2}^{2}}+x_{2}+x_{1}, \\
\frac{x_{2} x_{1}+x_{1} x_{2}+x_{1}^{2}}{x_{2}}-x_{1} x_{2} x_{1}-x_{1}^{3} \\
\frac{x_{1}^{5}}{x_{2} x_{1}^{2} x_{2}}+x_{2} x_{1}^{3}+\ldots \\
\frac{x_{2} x_{1}^{3} x_{2} x_{1}}{x_{2} x_{1}^{4} x_{2} x_{1}^{4}}+\ldots\end{array}$ & $\begin{array}{l}1, x_{1}, x_{2}, \\
x_{1}^{2}, x_{1} x_{2}, x_{2} x_{1}, \\
x_{1}^{3}, x_{1}^{2} x_{2}, \ldots, \\
x_{1}^{4}, \ldots, \\
\ldots\end{array}$ & 60 \\
\hline
\end{tabular}

Обозначим через $A_{\mathbb{G}}^{0} \in \mathbb{M}_{\mathbb{G}}$ алгебру с нильпотентными образующими, т. е.

$$
A_{\mathbb{G}}^{0}=\mathbb{C}\left\langle x_{1}, \ldots, x_{N} \mid x_{i}^{k_{i}}=0 \quad \forall i, \sum_{i=1}^{N} x_{i}=0\right\rangle .
$$

Из леммы 1 следует, что размерность (рост) алгебры $A_{\mathbb{G}}^{0}$ не меньше размерности (роста) любой другой алгебры в многообразии $\mathbb{M}_{\mathbb{G}}$.

Если граф $\mathbb{G}$ является диаграммой Дынкина, алгебра $A_{\mathbb{G}}^{0}$ оказывается конечномерной. Действительно, в табл. 1 приведены базисы Грёбнера для алгебр $A_{\mathbb{G}}^{0}$, которым соответствуют диаграммы Дынкина $\mathbb{G}$. Нетрудно выписать линейные базисы в этих алгебрах как множества всех мономов, не содержащих старших слов элементов базиса Грёбнера в качестве подслов. В первой колонке указан граф $\mathbb{G}$, вторая содержит соотношения алгебры $A_{\mathbb{G}}^{0}$ и выбранный порядок на образующих, третья - базис Грёбнера (старшие слова подчеркнуты, отточие обозначает сумму меньших слов с некоторыми коэффициентами), четвертая - нормальные слова алгебры $A_{\mathbb{G}}^{0}$ (мы перечисляем не все, если этих слов достаточно много), в пятой колонке указано количество нормальных слов, т. е. размерность алгебры $A_{\mathbb{G}}^{0}$. Тривиальный случай - две линейно связанные образующие с заданными спектрами - мы не приводим. Ему соответствуют диаграммы серии $A_{n}$. 
ТАБлицА 2. Алгебры полиномиального роста

\begin{tabular}{|c|c|c|c|c|}
\hline$\widetilde{D}_{4}$ & $\begin{array}{l}x_{1}^{2}=a_{11} x_{1}+a_{10}, \\
x_{2}^{2}=a_{21} x_{2}+a_{20}, \\
x_{3}^{2}=a_{31} x_{3}+a_{30}, \\
x_{4}^{2}=a_{41} x_{4}+a_{40} \\
x_{1}+x_{2}+x_{3}+x_{4}=0 \\
x_{4}>x_{3}>x_{2}>x_{1}\end{array}$ & $\begin{array}{l}\frac{x_{4}}{x_{1}^{2}}+x_{3}+x_{2}+x_{1}, \\
\frac{x_{2}^{2}}{x_{3}^{2}}+\ldots \\
\frac{x_{3}}{x_{2}}+\ldots \\
\frac{x_{3} x_{1}}{x_{2}}+\ldots\end{array}$ & 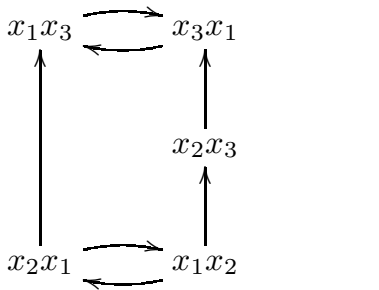 & {$\left[n^{2}\right]$} \\
\hline$\widetilde{E}_{6}$ & $\begin{array}{l}x_{1}^{3}=a_{12} x_{1}^{2}+a_{11} x_{1}+a_{10}, \\
x_{2}^{3}=a_{22} x_{2}^{2}+a_{21} x_{2}+a_{20}, \\
x_{3}^{3}=a_{32} x_{3}^{2}+a_{31} x_{3}+a_{30}, \\
x_{1}+x_{2}+x_{3}=0 ; \\
x_{3}>x_{2}>x_{1}\end{array}$ & $\begin{array}{l}\frac{x_{3}}{x_{1}^{3}}+x_{2}+x_{1}, \\
\frac{x_{2}^{3}}{x_{2}^{2}} x_{1}+\ldots, \\
\frac{x_{2} x_{2} x_{1} x_{2}+\ldots,}{x_{2} x_{1} x_{2} x_{1}^{2}}+\ldots\end{array}$ & $\begin{array}{c}x_{1}^{2} x_{2}^{2} \\
\uparrow \\
x_{2} x_{1}^{2} x_{2} \\
\mid \\
x_{1}^{2} x_{2} x_{1} \\
x_{1} x_{2} x_{1} x_{2} \\
\downarrow \\
x_{2} x_{1} x_{2}^{2}\end{array}$ & {$\left[n^{2}\right]$} \\
\hline
\end{tabular}

В случае когда $\mathbb{G}$ - расширенная диаграмма Дынкина, оказалось, что главные части элементов базиса Грёбнера не зависят от коэффициентов полиномов $P_{1}, \ldots, P_{N}$, т. е. одинаковы для всех алгебр, соответствующих данному графу. В табл. 2 и 3 приведены базисы Грёбнера и сосчитан рост алгебр, для которых граф $\mathbb{G}$ - расширенная диаграмма Дынкина. Здесь в первой колонке снова указан граф $\mathbb{G}$, вторая содержит соотношения алгебры (с неопределенными коэффициентами) и выбранный порядок на образующих, третья - базис Грёбнера, четвертая - граф роста, а в пятой колонке указан порядок роста алгебр, соответствующих $\mathbb{G}$. Оказалось, что в случае всех расширенных диаграмм Дынкина граф роста содержит ровно два цикла, соединенных путем, а значит, его рост квадратичен [3].

Если граф некоторой алгебры не является ни диаграммой Дынкина, ни расширенной диаграммой Дынкина, он обязательно содержит один из следующих графов: $T_{2,2,2,2,2}, T_{3,2,2,2}, T_{4,3,3}, T_{5,4,2}$ или $T_{7,3,2}$. Для этих графов старшие слова базисов Грёбнера соответствующих алгебр снова не зависят от коэффициентов полиномов $P_{1}, \ldots, P_{N}$. В табл. 4 мы указываем граф (первая колонка), соотношения соответствующих ему алгебр с неопределенными коэффициентами и выбранный нами порядок на образующих (вторая колонка), базис Грёбнера (третья колонка) и два такие слова $a$ и $b$, что все слова вида $a^{n_{1}} b^{m_{1}} a^{n_{2}} b^{m_{2}} \ldots$ $\left(n_{i}, m_{i} \geqslant 0\right)$ являются нормальными (последняя колонка). Тогда все алгебры, которым соответствуют перечисленные выше графы, содержат свободную подалгебру $\mathbb{C}\langle a, b\rangle$.

Пусть $K=\sum_{i=1}^{N} k_{i}$. Параметризуем семейство $\mathbb{M}_{\mathbb{G}}$ набором спектров образующих, т. е. вектору $a=\left(a_{1}, \ldots, a_{K}\right) \in \mathbb{C}^{K}$ поставим в соответствие алгебру $A_{P_{1}, \ldots, P_{N}}$ с $P_{1}(x)=\prod_{i=1}^{k_{1}}\left(x-a_{i}\right), P_{2}(x)=\prod_{i=k_{1}+1}^{k_{1}+k_{2}}\left(x-a_{i}\right)$ и т. д. 
ТАБлицА 3. Алгебры полиномиального роста (продолжение табл. 2)

\begin{tabular}{|c|c|c|c|c|}
\hline$\widetilde{E}_{7}$ & $\begin{aligned} & x_{1}^{4}= a_{13} x_{1}^{3}+a_{12} x_{1}^{2} \\
&+a_{11} x_{1}+a_{10}, \\
& x_{2}^{4}= a_{23} x_{2}^{3}+a_{22} x_{2}^{2} \\
&+a_{21} x_{2}+a_{20}, \\
& x_{3}^{2}= a_{31} x_{3}+a_{30}, \\
& x_{1}+ x_{2}+x_{3}=0 ; \\
& x_{3}>x_{2}>x_{1}\end{aligned}$ & $\begin{array}{l}\frac{x_{3}}{x_{1}^{4}}+x_{2}+x_{1}, \\
\frac{\frac{x_{2}^{2}}{2}+\ldots,}{x_{2} x_{1} x_{2} x_{1}} \\
\quad+x_{2} x_{1}^{2} x_{2}+\ldots, \\
\underline{x_{2} x_{1}^{2} x_{2} x_{1}^{3}}+\ldots\end{array}$ & 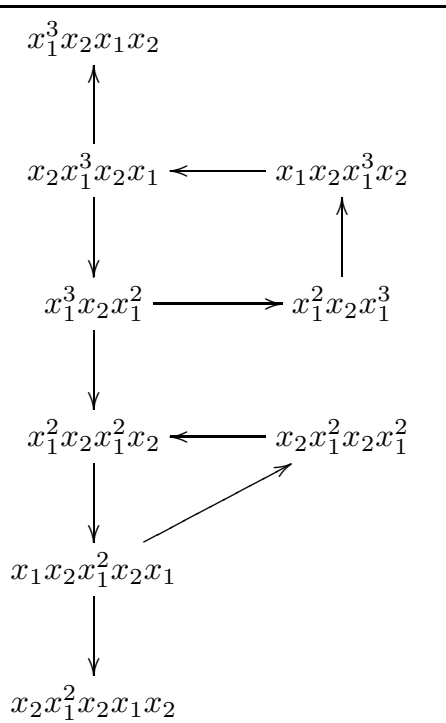 & {$\left[n^{2}\right]$} \\
\hline$\widetilde{E}_{8}$ & $\begin{aligned} & x_{1}^{6}= a_{15} x_{1}^{5}+a_{14} x_{1}^{4} \\
&+a_{13} x_{1}^{3}+a_{12} x_{1}^{2} \\
&+a_{11} x_{1}+a_{10}, \\
& x_{2}^{3}= a_{22} x_{2}^{2} \\
&+a_{21} x_{2}+a_{20}, \\
& x_{3}^{2}= a_{31} x_{3}+a_{30}, \\
& x_{1}+ x_{2}+x_{3}=0 ; \\
& x_{3}>x_{2}>x_{1}\end{aligned}$ & $\begin{array}{l}\frac{x_{3}}{x_{1}^{6}}+x_{2}+x_{1}, \\
\frac{x_{2}^{2}}{x_{2}} x_{1} x_{2}+\ldots, \\
\frac{x_{2} x_{1}^{2} x_{2}}{x_{2} x_{1}^{3} x_{2} x_{1}} \\
+x_{2} x_{1}^{4} x_{2}+\ldots, \\
\underline{x_{2} x_{1}^{4} x_{2} x_{1}^{5}}+\ldots\end{array}$ & $\left.\right|_{\uparrow} ^{x_{1}^{5} x_{2} x_{1}^{3} x_{2}}{ }_{\uparrow}^{x_{1}^{5} x_{2} x_{1}^{3}} x_{1} x_{2} x_{1}^{5} x_{2} x_{1}^{2}$ & {$\left[n^{2}\right]$} \\
\hline
\end{tabular}

Теперь докажем теорему 1.

ДокаЗАТЕЛЬСтво теоремы 1. Пусть $\mathbb{G}$ - диаграмма Дынкина и $A=$ $A_{P_{1}, \ldots, P_{N}} \in \mathbb{M}_{\mathbb{G}}$. Тогда, как показано выше, алгебра $A_{\mathbb{G}}^{0}$ конечномерна. Пусть 
ТАБлицА 4. Алгебры экспоненциального роста

\begin{tabular}{|c|c|c|c|}
\hline$T_{2,2,2,2,2}$ & $\begin{array}{l}x_{1}^{2}=a_{11} x_{1}+a_{10}, \\
x_{2}^{2}=a_{21} x_{2}+a_{20}, \\
x_{3}^{2}=a_{31} x_{3}+a_{30}, \\
x_{4}^{2}=a_{41} x_{4}+a_{40}, \\
x_{5}^{2}=a_{51} x_{5}+a_{50}, \\
x_{1}+x_{2}+x_{3}+x_{4}+x_{5}=0 \\
x_{5}>x_{4}>x_{3}>x_{2}>x_{1}\end{array}$ & $\begin{array}{l}\frac{x_{5}}{x_{1}^{2}}+x_{4}+x_{3}+x_{2}+x_{1}, \\
\frac{x_{2}^{2}}{\frac{x_{3}^{2}}{2}+\ldots} \\
\frac{x_{4}^{2}}{x_{4}}+\ldots \\
\frac{x_{3}}{x_{4} x_{2} x_{3}}+x_{4} x_{2}+\ldots, \\
\underline{x_{4}} x_{1} x_{3}+\ldots\end{array}$ & $\begin{array}{l}a=x_{1} x_{2}, \\
b=x_{1} x_{3}\end{array}$ \\
\hline$T_{3,2,2,2}$ & $\begin{array}{l}x_{1}^{3}=a_{12} x_{1}^{2}+a_{11} x_{1}+a_{10}, \\
x_{2}^{2}=a_{21} x_{2}+a_{20}, \\
x_{3}^{2}=a_{31} x_{3}+a_{30} \\
x_{4}^{2}=a_{41} x_{4}+a_{40} \\
x_{1}+x_{2}+x_{3}+x_{4}=0 \\
x_{4}>x_{3}>x_{2}>x_{1}\end{array}$ & $\begin{array}{l}\frac{x_{4}}{x_{1}^{3}}+x_{3}+x_{2}+x_{1}, \\
\frac{x_{2}^{2}}{2}+\ldots \\
\frac{x_{3}^{2}}{x_{3}} x_{2}+\ldots \\
\underline{x_{3} x_{1}} x_{2}-x_{3} x_{1}+\ldots, \\
\underline{2} x_{3} x_{1}+\ldots\end{array}$ & $\begin{array}{l}a=x_{1} x_{2} x_{1}, \\
b=x_{1} x_{3} x_{1}\end{array}$ \\
\hline$T_{4,3,3}$ & $\begin{array}{l}x_{1}^{4}=a_{13} x_{1}^{3}+a_{12} x_{1}^{2}+\ldots, \\
x_{2}^{3}=a_{22} x_{2}^{2}+a_{21} x_{2}+a_{20}, \\
x_{3}^{2}=a_{31} x_{3}+a_{30} \\
x_{1}+x_{2}+x_{3}=0 \\
x_{3}>x_{2}>x_{1}\end{array}$ & $\begin{array}{l}\frac{x_{3}}{x_{1}^{4}}+x_{2}+x_{1}, \\
\frac{x_{2}^{3}}{x_{2}^{2}} x_{1}+\ldots \\
\frac{x_{2} x_{1}}{x_{2} x_{1}^{3}}+\ldots \\
\end{array}$ & $\begin{array}{l}a=x_{1} x_{2}, \\
b=x_{1}^{2} x_{2}\end{array}$ \\
\hline$T_{5,4,2}$ & $\begin{array}{l}x_{1}^{5}=a_{14} x_{1}^{4}+a_{13} x_{1}^{3}+\ldots, \\
x_{2}^{4}=a_{23} x_{2}^{3}+a_{22} x_{2}^{2}+\ldots, \\
x_{3}^{2}=a_{31} x_{3}+a_{30} \\
x_{1}+x_{2}+x_{3}=0 \\
x_{3}>x_{2}>x_{1}\end{array}$ & $\begin{array}{l}\frac{x_{3}}{x_{1}^{5}}+x_{2}+x_{1}, \\
\frac{x_{2}^{2}}{\frac{x_{2}}{x_{1}} x_{2} x_{2} x_{1}}+x_{2} x_{1}+\ldots, \\
\frac{x_{2} x_{1}^{2} x_{2} x_{1}^{4}}{1}+\ldots\end{array}$ & $\begin{array}{l}a=x_{1}^{2} x_{2}, \\
b=x_{1}^{3} x_{2}\end{array}$ \\
\hline$T_{7,3,2}$ & $\begin{array}{l}x_{1}^{7}=a_{16} x_{1}^{6}+a_{15} x_{1}^{5}+\ldots, \\
x_{2}^{3}=a_{22} x_{2}^{2}+a_{21} x_{2}+a_{20}, \\
x_{3}^{2}=a_{31} x_{3}+a_{30} \\
x_{1}+x_{2}+x_{3}=0 \\
x_{3}>x_{2}>x_{1}\end{array}$ & $\begin{array}{l}\frac{x_{3}}{x_{1}^{7}}+x_{2}+x_{1}, \\
\frac{x_{2}^{2}}{x_{2}}+x_{1} x_{2} x_{1}+\ldots, \\
\frac{x_{2} x_{1}^{2} x_{2}}{x_{2} x_{1}^{3} x_{2} x_{1}}+x_{2} x_{1}+\ldots, \ldots, \\
\frac{x_{2} x_{1}^{4} x_{2} x_{1}^{6}}{2}+\ldots \\
\end{array}$ & $\begin{array}{l}a=x_{1}^{4} x_{2}, \\
b=x_{1}^{5} x_{2}\end{array}$ \\
\hline
\end{tabular}

$d^{0}=\operatorname{dim} A_{\mathbb{G}}^{0}$. Значения $d^{0}$ указаны в табл. 1. Из леммы 1 следует, что $d:=\operatorname{dim} A$ $\leqslant d^{0}$. Допустим, что алгебра $A$ ненулевая. Рассмотрим ее левое регулярное представление, и пусть комплексная матрица $X_{i}$ соответствует умножению на $x_{i}$ в алгебре $A$ для $i=1, \ldots, N$. Пусть $a_{1}^{i}, \ldots, a_{s_{i}}^{i}-$ корни полинома $P_{i}$ без учета кратности $\left(s_{i} \leqslant \operatorname{deg} P_{i}\right)$, и пусть $d_{j}^{i}-$ кратность собственного числа $a_{j}^{i}$ матрицы $X_{i}\left(j=1, \ldots, s_{i}\right)$. Тогда $\operatorname{tr} X_{i}=\sum_{j=1}^{s_{i}} a_{j}^{i} d_{j}^{i}$, и из равенства $\sum_{i=1}^{N} X_{i}=0$ мы получаем равенство для следов $\sum_{i=1}^{N} \sum_{j=1}^{s_{i}} a_{j}^{i} d_{j}^{i}=0$. Таким образом, вектор $\left(a_{1}^{1}, a_{2}^{1}, \ldots, a_{s_{N}}^{N}\right)$ ортогонален вектору $\left(d_{1}^{1}, d_{2}^{1}, \ldots, d_{s_{N}}^{N}\right)$ в пространстве $\mathbb{C}^{\sum_{i=1}^{N} s_{i}}$. 
Ясно, что вектор корней полиномов с учетом кратностей также ортогонален некоторому вектору с неотрицательными компонентами, сумма которых не превосходит $d_{0}$. Поскольку для данного графа $\mathbb{G}$ существует лишь конечное множество таких векторов, то существует такое конечное число гиперплоскостей в пространстве параметров $\mathbb{C}^{K}$, вне которого соответствующие алгебры нулевые.

Для расширенных диаграмм Дынкина мы уже проверили утверждение теоремы, построив графы роста в соответствующих алгебрах (они приведены в табл. 2 и 3$)$.

Третье утверждение теоремы докажем по индукции. Если граф $\mathbb{G}$ есть один из графов $T_{2,2,2,2,2}, T_{3,2,2,2}, T_{4,3,3}, T_{5,4,2}$ или $T_{7,3,2}$, то все алгебры семейства $\mathbb{M}_{\mathbb{G}}$ содержат свободную подалгебру с двумя образующими $\mathbb{C}\langle a, b\rangle$ (в табл. 4 указаны образующие $a$ и $b$ ). Любой другой граф, не являющийся ни диаграммой Дынкина, ни расширенной диаграммой Дынкина, можно получить из этих графов, удлиняя и приклеивая ветки. Пусть $\mathbb{G}=T_{k_{1}, \ldots, k_{N}}$ и $\mathbb{G}^{\prime}=T_{k_{1}-1, k_{2}, \ldots, k_{N}}$, если $k_{1}>2$, и $\mathbb{G}^{\prime}=T_{k_{2}, \ldots, k_{N}}$, если $k_{2}=2$. Пусть для $\mathbb{G}^{\prime}$ утверждение уже доказано. Рассмотрим произвольную алгебру $A \in \mathbb{M}_{\mathbb{G}}$,

$$
A=A_{P_{1}, \ldots, P_{N}}=\mathbb{C}\left\langle x_{1}, \ldots, x_{N} \mid P_{i}\left(x_{i}\right)=0 \forall i, \sum_{i=1}^{N} x_{i}=0\right\rangle,
$$

где $\operatorname{deg} P_{i}=k_{i}$ для $i=1, \ldots, N$. Пусть $a-$ корень полинома $P_{1}$ и $Q(x)=$ $P_{1}(x) /(x-a)$. Тогда факторалгебра $A^{\prime}=A /\left\{Q\left(x_{1}\right)=0\right\}$ принадлежит семейству $\mathbb{M}_{\mathbb{G}^{\prime}}$. По предположению индукции $A^{\prime}$ содержит свободную подалгебру с двумя образующими. Тогда то же самое верно и для $A$, что и требовалось доказать.

\section{§3. Центральные элементы и полиномиальные тождества}

Рассмотрим семейство алгебр, которым соответствуют расширенные диаграммы Дынкина. Свойство таких алгебр быть PI-алгебрами зависит от самих полиномов, а не только от их степеней. Пусть $S=S\left(P_{1}, \ldots, P_{N}\right)$ задается равенством $S=\sum_{i=1}^{N} s_{i}$, где $s_{i}$ есть среднее арифметическое корней полинома $P_{i}$ с учетом их кратностей.

Для случая диаграммы $\widetilde{D}_{4}$ из работ [6,7] следует, что при $S=0$ алгебра $A$ является $\mathbb{F}_{4}$-алгеброй. Для $\widetilde{E}_{6}$ в [8] с помощью описания *-представлений алгебры $A_{P_{1}, P_{2}, P_{3}}$ из работы [9], показано, что при $P_{1}(x)=P_{2}(x)=P_{3}(x)=$ $(x-1) x(x+1)$ эта алгебра является $\mathbb{F}_{6}$-алгеброй.

В этом параграфе мы доказываем следующую теорему:

Теорема 2. Алгебра $A=A_{P_{1}, \ldots, P_{N}}$, для которой граф $\mathbb{G}$ есть расширенная диаграмма Дынкина и $S=0$, является конечно порожденным модулем над своим центром и, следовательно, алгеброй с полиномиальными тождествами.

Приведенное ниже доказательство теоремы исчерпывает случаи $\widetilde{E}_{6}, \widetilde{E}_{7}$ и $\widetilde{E}_{8}$. Для случая $\widetilde{D}_{4}$ существует аналогичное доказательство, но мы его здесь не приводим.

Для начала заметим, что в случае $S=0$ мы можем перейти к новой системе образующих $x_{i}^{\prime}=x_{i}-s_{i}, i=1, \ldots, N$, где $s_{i}$ - среднее арифметическое корней 
полинома $P_{i}$. Тогда соотношения перепишутся следующим образом:

$$
P_{i}^{\prime}\left(x_{i}^{\prime}\right)=0, \quad \sum_{i=1}^{N} x_{i}^{\prime}=0,
$$

где у полиномов $P_{i}^{\prime}$ коэффициент при члене степени $\operatorname{deg} P_{i}^{\prime}-1$ уже равен нулю. Поэтому без ограничения общности можно предположить, что у исходных полиномов $P_{i}$ коэффициент при члене степени $\operatorname{deg} P_{i}-1$ равен нулю. Также будем предполагать, что $\operatorname{deg} P_{1} \geqslant \operatorname{deg} P_{2} \geqslant \operatorname{deg} P_{3}$ (в случае расширенных диаграмм Дынкина $N=3)$. Это означает, что $\left(\operatorname{deg} P_{1}, \operatorname{deg} P_{2}, \operatorname{deg} P_{3}\right)$ - одна из троек $(3,3,3),(4,4,2),(6,3,2)$.

Сначала мы найдем два элемента $z_{1}, z_{2}$ из центра алгебры $A$. Оказывается, их будет достаточно для того, чтобы алгебра $A$ была конечно порожденным модулем над $\mathbb{C}\left(z_{1}, z_{2}\right)$. Для нахождения элементов центра введем следующие обозначения. Пусть

$$
\left\{v_{1}, \ldots, v_{n}\right\}=\sum_{\pi \in S_{n}} v_{\pi(1)} \cdots v_{\pi(n)},
$$

где $v_{1}, \ldots, v_{n}$ принадлежат алгебре $A$, и пусть $\left\{v^{k}: w^{l}\right\}-$ сумма всевозможных слов из $k$ букв $v$ и $l$ букв $w$. Если $P(x)=\sum_{i=0}^{n} p_{i} x^{i}-$ некоторый полином, то положим

$$
P(v: w)=\sum_{i=1}^{n} p_{i}\left\{v^{i-1}: w\right\} .
$$

Лемма 2. Если $v \in A$ и $P$ - полином, аннулирующий $v$, mо $P(v: w)$ коммутирует с $v$ для любого $w \in A$.

ДокАзАТЕЛьство. Раскрывая скобки, можно убедиться, что

$$
[P(v: w), v]=[w, P(v)]=0 .
$$

Теперь опишем процедуру построения элементов из центра. Пусть $L_{0} \subset A-$ линейное пространство, состоящее из элементов, которые представляются в виде суммы двух элементов, из которых один является полиномом от $x_{1}$, а другой коммутирует с $x_{2}$. Когда будет определен первый элемент из центра, $z_{1}$, то символом $L_{1}$ мы будем обозначать пространство элементов, которые представляются в виде суммы двух, из которых один - полином над $\mathbb{C}\left(z_{1}\right)$ от $x_{1}$, а другой коммутирует с $x_{2}$. Допустим теперь, что $P_{1}\left(x_{1}: y_{1}\right)$ лежит в $L_{0}$ для некоторого $y_{1} \in A$. Это значит, что из $P_{1}\left(x_{1}: y_{1}\right)$ можно вычесть некоторый полином от $x_{1}$ и получить элемент из центра. Обозначим этот элемент символом $z_{1}$. Теперь пусть $P_{1}\left(x_{1}: y_{2}\right)$ лежит в $L_{1}$ для некоторого $y_{2} \in A$. Аналогично, из $P_{1}\left(x_{1}: y_{2}\right)$ можно вычесть полином от $x_{1}$ (только теперь над $\mathbb{C}\left(z_{1}\right)$ ) и получить второй элемент из центра, $z_{2}$. Положим

$$
y_{1}=x_{2},
$$

ПРЕДЛОЖЕНИЕ 2. Элемент $P_{1}\left(x_{1}: x_{2}\right)$ лежит в $L_{0}=\mathbb{C}\left(x_{1}\right)+Z\left(x_{2}\right)$, где $Z\left(x_{2}\right)$ - пространство элементов, коммутирующих с $x_{2}$.

ДокАЗАТЕЛЬство. По определению $P_{1}\left(x_{1}: x_{2}\right)$ является линейной комбинацией элементов вида $\left\{x_{1}^{k}: x_{2}\right\}$ с $k \leqslant \operatorname{deg} P_{1}-1$, причем так как $P_{1}(x)$ не содержит $x^{\operatorname{deg} P_{1}-1}$, то в эту линейную комбинацию не входит элемент с $k=\operatorname{deg} P_{1}-2$. 
Мы покажем, что каждый из элементов вида $\left\{x_{1}^{k}: x_{2}\right\}$, входящих в разложение элемента $P_{1}\left(x_{1}: x_{2}\right)$, лежит в $L_{0}$. Рассмотрим такие пары неотрицательных целых чисел $(k, l)$, что $\left\{x_{1}^{k}: x_{2}^{l}\right\}$ лежит в $L_{0}$.

1. Если $k=0$ или $l=0$, то $\left\{x_{1}^{k}: x_{2}^{l}\right\}$ лежит в $L_{0}$. Это непосредственно следует из определения.

2. $x_{3}^{k} \in L_{0}$ для любого $k$ в случаях $\widetilde{E}_{7}$ и $\widetilde{E}_{8}$ и для $k=1,3$ в случае $\widetilde{E}_{6}$. Так как в случаях $\widetilde{E}_{7}$ и $\widetilde{E}_{8}$ степень полинома $P_{3}$ равна двум, $x_{3}^{k}$ является линейной комбинацией единицы и $x_{3}=-x_{1}-x_{2}$, которые лежат в $L_{0}$. В случае $\widetilde{E}_{6}$ это также верно для $k=3$ и $k=1$, так как $P_{3}-$ кубический полином без квадратов.

3. В случаях $\widetilde{E}_{7}$ и $\widetilde{E}_{8}$ элемент $\left\{x_{1}: x_{2}\right\}$ лежит в $L_{0}$, так как

$$
\left\{x_{1}: x_{2}\right\}=x_{3}^{2}-x_{1}^{2}-x_{2}^{2} .
$$

4. $\left\{x_{1}: x_{2}^{k}\right\} \in L_{0}$ для любого $k$ в случае $\widetilde{E}_{8}$, для $k=0,1,3$ в случае $\widetilde{E}_{7}$ и для $k=0,2$ в случае $\widetilde{E}_{6}$. Для $\widetilde{E}_{8}$ представим $x_{2}^{k+1}$ как линейную комбинацию элементов $x_{2}^{2}, x_{2}$ и $е$. Пусть

$$
x_{2}^{k+1}-a_{2} x_{2}^{2}-a_{1} x_{2}-a_{0}=0, \quad \text { где } a_{0}, a_{1}, a_{2} \in \mathbb{C} .
$$

Воспользовавшись леммой 2 , получаем, что

$$
\left\{x_{1}: x_{2}^{k}\right\}-a_{2}\left\{x_{1}: x_{2}\right\}-a_{1} x_{1} \text { коммутирует с } x_{2},
$$

и это завершает доказательство. В случаях $\widetilde{E}_{6}$ и $\widetilde{E}_{7}$ аналогичным образом нужно выразить $x_{2}^{3}$ через $x_{2}$ и $e$ и $x_{2}^{4}$ через $x_{2}^{2}, x_{2}$ и $e$, а затем воспользоваться такими же соображениями.

5. Для случаев $\widetilde{E}_{6}$ и $\widetilde{E}_{8}$ элемент $\left\{x_{1}^{2}: x_{2}\right\}$ лежит в $L_{0}$. Это следует из тождества

$$
-x_{3}^{3}=\left(x_{1}+x_{2}\right)^{3}=x_{1}^{3}+\left\{x_{1}^{2}: x_{2}\right\}+\left\{x_{1}: x_{2}^{2}\right\}+x_{2}^{3},
$$

и из предыдущих пунктов. Для $\widetilde{E}_{6}$ элемент $P_{1}\left(x_{1}: x_{2}\right)$, являясь линейной комбинацией элементов $\left\{x_{1}^{2}: x_{2}\right\}$ и $\left\{x_{1}^{0}: x_{2}\right\}$, лежит в $L_{0}$, и доказательство для этого случая завершено.

6. Для случаев $\widetilde{E}_{7}$ и $\widetilde{E}_{8}$ элемент $\left\{x_{1}^{3}: x_{2}\right\}$ принадлежит $L_{0}$. Можно воспользоваться тем, что

$$
\begin{aligned}
\left\{x_{3}^{2}, x_{1}^{2}-x_{2}^{2}\right\} & =\left\{x_{1}^{2}+x_{2}^{2}, x_{1}^{2}-x_{2}^{2}\right\}+\left\{\left\{x_{1}, x_{2}\right\}, x_{1}^{2}-x_{2}^{2}\right\} \\
& =2 x_{1}^{4}-2 x_{2}^{4}+\left\{x_{1}^{3}: x_{2}\right\}-\left\{x_{1}: x_{2}^{3}\right\}
\end{aligned}
$$

причем так как $x_{3}^{2}$ кратен единице, левая часть принадлежит $L_{0}$. Кроме того, $x_{1}^{4}, x_{2}^{4}$ и $\left\{x_{1}: x_{2}^{3}\right\}$ принадлежат $L_{0}$. Значит, и $\left\{x_{1}^{3}: x_{2}\right\}$ принадлежит $L_{0}$. Для случая $\widetilde{E}_{7}$ это завершает доказательство.

7. Осталось разобрать случай $\widetilde{E}_{8}$ и доказать, что $\left\{x_{1}^{5}: x_{2}\right\}$ принадлежит $L_{0}$, Пусть $P_{2}(x)=x^{3}-b_{1} x-b_{0}$. Сначала заметим, что

$$
\begin{aligned}
\left\{x_{1}^{2}: x_{2}^{4}\right\} & =\left\{\left\{x_{1}^{2}: x_{2}\right\}, x_{2}^{3}\right\}+\left(\left\{x_{1}: x_{2}^{2}\right\}\right)^{2} \\
& =b_{1}\left\{\left\{x_{1}^{2}: x_{2}\right\}, x_{2}\right\}+2 b_{0}\left\{x_{1}^{2}: x_{2}\right\}+\left(\left\{x_{1}: x_{2}^{2}\right\}-b_{1} x_{1}+b_{1} x_{1}\right)^{2} .
\end{aligned}
$$

Учитывая то, что $\left\{x_{1}: x_{2}^{2}\right\}-b_{1} x_{1}$ коммутирует с $x_{2}$, получаем, что последнее выражение эквивалентно $\left(\bmod L_{0}\right)$ выражению

$$
b_{1}\left(\left\{\left\{x_{1}^{2}: x_{2}\right\}, x_{2}\right\}+\left\{\left\{x_{1}: x_{2}^{2}\right\}, x_{1}\right\}\right)=2 b_{1}\left\{x_{1}^{2}: x_{2}^{2}\right\} \text {. }
$$


Так как

$$
x_{3}^{4}=\left(x_{1}+x_{2}\right)^{4}=x_{1}^{4}+\left\{x_{1}^{3}: x_{2}\right\}+\left\{x_{1}^{2}: x_{2}^{2}\right\}+\left\{x_{1}: x_{2}^{3}\right\}+x_{2}^{4},
$$

из того, что $x_{3}^{4}, x_{1}^{4},\left\{x_{1}^{3}: x_{2}\right\},\left\{x_{1}: x_{2}^{3}\right\}$ и $x_{2}^{4}$ лежат в $L_{0}$, следует, что $\left\{x_{1}^{2}: x_{2}^{2}\right\}, \mathrm{a}$ значит, и $\left\{x_{1}^{2}: x_{2}^{4}\right\}$ лежат в $L_{0}$.

Пусть $P_{3}(x)=x^{2}-c_{0}$, т. е. $x_{3}^{2}=c_{0}$. Рассмотрим следующие равенства:

$$
\begin{gathered}
\frac{\left\{x_{2}^{2}, x_{2}^{2},\left\{x_{1}, x_{2}\right\}\right\}}{=}=2\left\{x_{1}: x_{2}^{5}\right\}, \\
\left\{x_{2}^{2}, x_{2}^{2}, x_{2}^{2}\right\}+\left\{x_{2}^{2}, x_{2}^{2},\left\{x_{1}, x_{2}\right\}\right\}+\underline{\left\{x_{2}^{2}, x_{2}^{2}, x_{1}^{2}\right\}}=\left\{x_{2}^{2}, x_{2}^{2}, x_{3}^{2}\right\}=6 c_{0} x_{2}^{4}, \\
\left\{x_{2}^{2}, x_{2}^{2}, x_{1}^{2}\right\}+\underline{\left\{x_{2}^{2},\left\{x_{1}, x_{2}\right\},\left\{x_{1}, x_{2}\right\}\right\}}=2\left\{x_{1}^{2}: x_{2}^{4}\right\}, \\
\left\{x_{2}^{2},\left\{x_{1}, x_{2}\right\}, x_{2}^{2}\right\}+\left\{x_{2}^{2},\left\{x_{1}, x_{2}\right\},\left\{x_{1}, x_{2}\right\}\right\}+\underline{\left\{x_{2}^{2},\left\{x_{1}, x_{2}\right\}, x_{1}^{2}\right\}} \\
=\left\{x_{2}^{2},\left\{x_{1}, x_{2}\right\}, x_{3}^{2}\right\}=3 c_{0}\left\{x_{2}^{2},\left\{x_{1}, x_{2}\right\}\right\}=3 c_{0}\left\{x_{1}: x_{2}^{3}\right\}, \\
\left\{x_{1}^{2}, x_{2}^{2}, x_{2}^{2}\right\}+\left\{x_{1}^{2}, x_{2}^{2},\left\{x_{1}, x_{2}\right\}\right\}+\underline{\left\{x_{1}^{2}, x_{2}^{2}, x_{1}^{2}\right\}} \\
=\left\{x_{1}^{2}, x_{2}^{2}, x_{3}^{2}\right\}=3 c_{0}\left\{x_{1}^{2}, x_{2}^{2}\right\}=3 c_{0}\left(2 c_{0} x_{2}^{2}-2 x_{2}^{4}-\left\{x_{1}: x_{2}^{3}\right\}\right), \\
\left\{x_{1}^{2}, x_{1}^{2}, x_{2}^{2}\right\}+\left\{x_{1}^{2}, x_{1}^{2},\left\{x_{1}, x_{2}\right\}\right\}+\left\{x_{1}^{2}, x_{1}^{2}, x_{1}^{2}\right\}=\left\{x_{1}^{2}, x_{1}^{2}, x_{3}^{2}\right\}=6 c_{0} x_{1}^{4}, \\
\left\{x_{1}^{2}, x_{1}^{2},\left\{x_{1}, x_{2}\right\}\right\}=2\left\{x_{1}^{5}: x_{2}\right\} .
\end{gathered}
$$

Используя эти равенства последовательно, получаем, что подчеркнутые выражения принадлежат $L_{0}$. Это завершает доказательство для $\widetilde{E}_{8}$.

Итак, пусть $P_{1}\left(x_{1}: x_{2}\right)=Q_{1}\left(x_{1}\right)+z_{1}$, где $Q_{1}-$ некоторый полином, а $z_{1}$ коммутирует с $x_{2}$. Тогда $z_{1}$ коммутирует с $x_{1}$ в силу леммы 2 и, значит, лежит в центре.

ПрЕДЛОЖЕНИЕ 3. Элемент $P_{1}\left(x_{1}: y_{2}\right)$ лежит в $L_{1}=\mathbb{C}\left(x_{1}, z_{1}\right)+Z\left(x_{2}\right)$, где $Z\left(x_{2}\right)$ - пространство элементов, коммутирующих с $x_{2}$, а $y_{2}$ задано формулой

$$
y_{2}= \begin{cases}x_{2}^{2} & \text { для случая } \widetilde{E}_{6}, \\ x_{2} x_{1} x_{2} & \text { для случая } \widetilde{E}_{7}, \\ x_{2} x_{1}^{3} x_{2} & \text { для случая } \widetilde{E}_{8} .\end{cases}
$$

Доказательство. Это утверждение эквивалентно тому, что $\left[P_{1}\left(x_{1}: y_{2}\right), x_{2}\right]$ является линейной комбинацией элементов вида $\left[P_{1}\left(x_{1}: x_{2}\right)^{k} x_{1}^{l}, x_{2}\right]$, так как $\mathbb{C}\left(x_{1}, z_{1}\right)=\mathbb{C}\left(x_{1}, P_{1}\left(x_{1}: x_{2}\right)\right)$, а $P_{1}\left(x_{1}: x_{2}\right)^{k} x_{1}^{l}$ образуют базис в $\mathbb{C}\left(x_{1}, P_{1}\left(x_{1}: x_{2}\right)\right)$. Оказывается, требуемую линейную комбинацию можно составить из элементов $\left[P_{1}\left(x_{1}: x_{2}\right)^{k} x_{1}^{l}, x_{2}\right]$ для $k=0,1$ и $l=1, \ldots, \operatorname{deg} P_{1}-1$. Мы проверили это утверждение с помощью написанной вторым автором программы для вычислений в конечно заданных алгебрах, которая основана на технике базисов Грёбнера. Эта программа доступна в Интернете по адресу http ://www . imath. kiev .ua/ mellit /groebner.

Теперь $P_{1}\left(x_{1}: y_{2}\right)=Q_{2}\left(x_{1}, z_{1}\right)+z_{2}$, где $Q_{2}-$ некоторый полином от двух переменных, а $z_{2}$ коммутирует с $x_{2}$. Следовательно, $z_{2}$ лежит в центре. Рассмотрим подалгебру $B=\mathbb{C}\left(z_{1}, z_{2}\right)$, лежащую в центре алгебры $A$. Справедливо следующее утверждение:

ПРЕДЛОЖЕНИЕ 4. Алгебра А является конечно порожденным модулем над $B$. 
Доказательство. Рассмотрим на словах из букв $x_{1}, x_{2}, x_{3}$ порядок, заданный в $\S 1$. Назовем слово $w$ нормальным относительно $B$, если оно не является линейной комбинацией над $B$ слов, строго меньших $w$. Можно доказать следующие свойства нормальных относительно $B$ слов:

(1) нормальные слова над $B$ порождают $A$ как $B$-модуль;

(2) любое подслово нормального относительно $B$ слова также нормально относительно $B$;

(3) множество нормальных относительно $B$ слов содержится в множестве нормальных слов (см. §1).

Докажем, что нормальных относительно $B$ слов не может быть бесконечно много. Рассмотрим равенства

$$
P_{1}\left(x_{1}: x_{2}\right)=Q_{1}\left(x_{1}\right)+z_{1}, \quad P_{1}\left(x_{1}: y_{2}\right)=Q_{2}\left(x_{1}, z_{1}\right)+z_{2} .
$$

Так как $P_{1}\left(x_{1}\right)=0$, мы можем преобразовать $Q_{1}\left(x_{1}\right)$ и $Q_{2}\left(x_{1}, z_{1}\right)$ так, чтобы их степень как полиномов от переменной $x_{1}$ была меньше степени полинома $P_{1}$. Найдем старшие слова элементов $P_{1}\left(x_{1}: x_{2}\right)$ и $P_{1}\left(x_{1}: y_{2}\right)$, оставшиеся после редукции с помощью базиса Грёбнера, приведенного в табл. 2 и 3. Отточиями мы обозначаем младшие слова, если они не повлияют на дальнейшие выкладки. Старшие слова подчеркнуты. Имеется 3 случая:

(1) Случай $\widetilde{E}_{6}$ :

$$
P_{1}\left(x_{1}: x_{2}\right)=\underline{x_{2} x_{1}^{2}}+\cdots .
$$

Здесь старшее слово уже нормально и поэтому редукции не требуется. Далее,

$$
P_{1}\left(x_{1}: y_{2}\right)=P_{1}\left(x_{1}: x_{2}^{2}\right)=x_{2}^{2} x_{1}^{2}+x_{1} x_{2}^{2} x_{1}+\cdots .
$$

Мы применяем редукцию относительно элемента базиса Грёбнера

$$
x_{2}^{2} x_{1}+x_{2} x_{1} x_{2}+\cdots
$$

и получаем, что элемент $P_{1}\left(x_{1}: y_{2}\right)$ эквивалентен следующему:

$\left(x_{2}^{2} x_{1}^{2}+x_{1} x_{2}^{2} x_{1}+\cdots\right)-\left(x_{2}^{2} x_{1}+x_{2} x_{1} x_{2}+\cdots\right) x_{1}=\left(x_{1} x_{2}^{2} x_{1}+\cdots\right)-\left(x_{2} x_{1} x_{2} x_{1}+\cdots\right)$.

Так как все слова в выражении $x_{1} x_{2}^{2} x_{1}+\cdots$ меньше $x_{2} x_{1} x_{2} x_{1}$, это выражение можно отбросить, и получается, что старшим словом в результате оказывается $x_{2} x_{1} x_{2} x_{1}$ с коэффициентом -1. Аналогично разбираются остальные случаи.

(2) Случай $\widetilde{E}_{7}$ :

$$
\begin{gathered}
P_{1}\left(x_{1}: x_{2}\right)=\underline{x_{2} x_{1}^{3}}+\cdots, \\
P_{1}\left(x_{1}: y_{2}\right)=P_{1}\left(x_{1}: x_{2} x_{1} x_{2}\right)=x_{2} x_{1} x_{2} x_{1}^{3}+x_{1} x_{2} x_{1} x_{2} x_{1}^{2}+\cdots=\underline{-x_{2} x_{1}^{2} x_{2} x_{1}^{2}}+\cdots .
\end{gathered}
$$

(3) Случай $\widetilde{E}_{8}$ :

$$
\begin{gathered}
P_{1}\left(x_{1}: x_{2}\right)=\underline{x_{2} x_{1}^{5}}+\cdots, \\
P_{1}\left(x_{1}: y_{2}\right)=P_{1}\left(x_{1}: x_{2} x_{1}^{3} x_{2}\right)=x_{2} x_{1}^{3} x_{2} x_{1}^{5}+x_{1} x_{2} x_{1}^{3} x_{2} x_{1}^{4}+\cdots=\underline{-x_{2} x_{1}^{4} x_{2} x_{1}^{4}+\cdots .}
\end{gathered}
$$

Заметим, что найденные старшие слова выражаются с помощью формул (3.1) через меньшие слова с коэффициентами из $B$, так как степени $Q_{1}\left(x_{1}\right)$ и $Q_{2}\left(x_{1}, z_{1}\right)$ как полиномов от $x_{1}$ над $B$ меньше длин найденных старших слов в каждом из трех случаев. Это значит, что эти слова не являются нормальными относительно $B$. Рассмотрим графы роста алгебры $A$, приведенные в табл. 2 и 3. Следующие слова не являются нормальными относительно $B$ : 
(1) $x_{2} x_{1}^{2} x_{2}, x_{1} x_{2} x_{1}^{2}, x_{2} x_{1} x_{2} x_{1}$ для $\widetilde{E}_{6}$,

(2) $x_{2} x_{1}^{3} x_{2} x_{1}, x_{1} x_{2} x_{1}^{3} x_{2}, x_{1}^{2} x_{2} x_{1}^{3}, x_{2} x_{1}^{2} x_{2} x_{1}^{2}$ для $\widetilde{E}_{7}$,

(3) $x_{2} x_{1}^{5} x_{2} x_{1}^{3}, x_{1} x_{2} x_{1}^{5} x_{2} x_{1}^{2}, x_{1}^{2} x_{2} x_{1}^{5} x_{2} x_{1}, x_{1}^{3} x_{2} x_{1}^{5} x_{2}, x_{1}^{4} x_{2} x_{1}^{5}, x_{2} x_{1}^{4} x_{2} x_{1}^{4}$ для $\widetilde{E}_{8}$. Значит, если мы вычеркнем эти слова из графа роста, то оставшиеся пути будут исчерпывать все достаточно длинные нормальные относительно $B$ слова. Из того, что количество оставшихся путей конечно, следует конечность множества нормальных относительно $B$ слов, а значит, и тот факт, что алгебра $A$ является конечно порожденной как модуль над $B$.

\section{ЛитерАТУРА}

1. Fulton $W$. Eigenvalues, invariant factors, highest weights, and Schubert calculus. Bull. Amer. Math. Soc., 37, No. 3, 209-249 (2000).

2. Klyachko A. A. Stable bundles, representation theory and Hermitian operators. Selecta Math., 4, 419-445 (1998).

3. Уфнаровский B. А. Комбинаторные и асимптотические методы в алгебре. В сб.: Итоги науки и техники. Современные проблемы математики. Фундаментальные направления. Т. 57, 1990, с. 5-177.

4. Rowen L. H. Ring theory. Academic Press, 1991.

5. Пирс P. Ассоциативные алгебры. Мир, М., 1986.

6. Рабанович В. И., Самойленко Ю. С., Стрелеи А. В. О тождествах в алгебрах $Q_{n, \lambda}$, порожденных идемпотентами. Укр. матем. ж., 53, № 10, 1380-1390 (2001).

7. Рабанович В. И., Самойленко Ю. С., Стрелеи, А. В. О тождествах в алгебрах, порожденных линейно связанными идемпотентами. Укр. матем. ж., 56, №6, 782795 (2004).

8. Strelets $A . V$. On identities in the algebra generated by three partial reflections sum of which is zero. Methods Funct. Anal. Topol., 10, No. 2, 86-90 (2004).

9. Меллит A. C. Когда сумма трех частичных отражений равна нулю. Укр. матем. ж., 55, №9, 1277-1283 (2003).

10. Crawley-Boevey W., Holland M. P. Noncommutative deformations of Kleinian singularities. Duke Math. J., 92, No. 3, 605-635 (1998).

11. Ringel $C$. M. The preprojective algebra of a quiver. In: Algebras and modules, II, CMS Conf. Proc., Vol. 24, Amer. Math. Soc., Providence, RI, 1998, pp. 467-480.

12. Гельфанд И. М., Пономарев В. А. Модельные алгебры и представления графов. Функц. анализ и его прил., 13, вып. 3, 1-12 (1979).

13. Рабанович В. И., Самойленко Ю. С. Когда сумма идемпотентов или проекторов кратна единице. Функц. анализ и его прил., 34, вып. 4, 91-93 (2000).

14. Кругляк С. А., Рабанович В. И., Самойленко Ю. С. О суммах проекторов. Функц. анализ и его прил., 36, вып. 3, 20-35 (2002).

15. Кругляк C. A., Ройтер A. В. Локально-скалярные представления графов в категории гильбертовых пространств. Препринт Института математики НАН Украины, 2003.4, 2003.

Институт математики НАН Украины, e-mail: mariyka@imath.kiev.ua

Поступило в редакцию 12 февраля 2003 г.

Институт математики НАН Украины, e-mail: mellit@imath.kiev.ua

Институт математики НАН Украины, e-mail: yurii_sam@imath.kiev.ua 